\title{
具有长波吸收的(焦)脱镁叶绿酸衍生物的合成及其电子光谱
}

\author{
杨 泽 ${ }^{a}$ 王 振 ${ }^{a}$ 刘 洋 ${ }^{a}$ 徐希森 ${ }^{a}$ 祁彩霞 ${ }^{b}$ 王进军 $*, a, b$

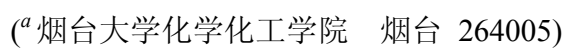 \\ ( ${ }^{b}$ 山东省黄金工程技术研究中心(工业应用) 烟台 264005)
}

\begin{abstract}
摘要 以脱镁叶绿酸-a 甲酯和脱镁叶绿酸-b 甲酯为起始原料, 通过 3-位乙烯基、外接 E-环和 20-meso-位的化学修饰, 在 脱镁叶绿酸-a 甲酯周环上建立了不同的羰基结构. 利用脱镁叶绿酸-a 甲酯的所建羰基和脱镁叶绿酸-b 甲酯的原有 7-位 甲酰基分别与丙二睛进行 Knoevenagel 缩合反应, 在二氢卟吩的不同位置引进了 $\beta, \beta$-二氧亚甲基结构, 完成一系列具有 长波吸收的叶绿素类二氢卟吩衍生物的合成, 讨论了 $\beta, \beta$-二氧亚甲基的引入对大环分子的化学活性和电子光谱的影响, 并对相应的化学反应提出可能的反应机理. 未见报道的 10 个叶绿素-a 衍生物均经 UV, IR, ${ }^{1} \mathrm{H}$ NMR 及元素分析证明其 结构.
\end{abstract}

关键词 叶绿素; (焦)脱镁叶绿酸; 二氢卟吩; 合成; 电子光谱

\section{Synthesis of (Pyro)pheophorbide Derivatives with Long Wave- length Absorption and Their Electronic Spectra}

\author{
Yang, Ze ${ }^{a} \quad$ Wang, Zhen ${ }^{a} \quad$ Liu, Yang $^{a} \quad$ Xu, Xisen ${ }^{a} \quad$ Qi, Caixia $^{b} \quad$ Wang, Jinjun*,a,b \\ ( ${ }^{a}$ College of Chemistry and Chemical Engineering, Yantai University, Yantai 264005) \\ ( ${ }^{b}$ Shandong Applied Research Centre of Gold Nanotechnology (Au-SDARC), Yantai 264005)
}

\begin{abstract}
Methyl pheophorbide-a and b were used as starting material. The different carbonyl groups were built along the periphery of methyl pheophorbide-a by modifing for $\mathrm{C}(3)$-vinyl group, exocyclic E-ring and 20-meso-position. Making use of these carbonyl groups or original $\mathrm{C}(7)$-formyl group of pheophorbide-b introduced $\beta, \beta$-dicyanomethylene moiety at different positions by Knoevenagel condensation with malononitrile to synthesize a series of chlorophyllous chlorin derivatives with long wavelength absorption. The effect on the electronic spectra and chemical activities of macrocycle molecule due to introduction of $\beta, \beta$-dicyanomethylene moiety were discussed, and the possible mechanisms about corresponding reactions were tentatively proposed. The structures of all new chlorin derivatives were characterized by ${ }^{1} \mathrm{H}$ NMR, UV, IR spectra and elemental analysis.
\end{abstract}

Keywords chlorophyll; (pyro)pheophorbide; chlorin; synthesis; electronic spectra

尽管天然细菌叶绿素及其人工合成同系物的光物 理性质可以完全满足光动力治疗(Photodynamic therapy, 简称 PDT)的基本要求, 同时在药理试验和临床应用中 也显示出高效的光动力抗癌活性, 但其细菌二氢卟吩 (四氢卟吩)大环结构的光热稳定性极差, 对体内自然的 生理环境相当敏感, 在治疗过程中非常容易转化成相对 稳定的二氢卟吩环系, 分子的最大可见光吸收(Qy 吸收) 也随之发生大幅度蓝移 ${ }^{[1 \sim 3]}$. 二氢卟吩碳架的形成减损 了光敏剂对生物组织的穿透能力, 所导致的光热敏感度
和光毒活性的降低将直接影响着 PDT 疗效, 因而使得 细菌二氢卟吩类光敏剂在光动力治疗的应用中受到了 严重限制. 因此, 如何获取稳定的长波光敏剂( $\mathrm{Qy}>700$ $\mathrm{nm})$ 一直是光动力治疗中的重要研究内容 ${ }^{[4 \sim 6]}$, 而叶绿 素衍生物的非对称性化学结构、多取代活性官能团和稳 定的光敏性质则是制备理想 PDT 光敏剂的良好基础条 件. 在前期的叶绿素化学的研究中, 我们通过对叶绿 素-a 降解产物的结构修饰, 合成出大量的具有叶绿素基 本碳架的新型二氢卟吩衍生物, 其中, 许多四吡咯大环

*E-mail: wjj1955@163.com

Received September 6, 2012; revised September 23, 2012; published online October 11, 2012.

Project supported by the Technology Cooperation between Governments of China and Hungary and the Shandong Applied Research Centre of Gold Nanotechnology (2011).

中洶政府间科技合作(No. 2008-333-4-32)和山东黄金工程技术研究中心(2011 年度)资助项目. 
化合物已经表现出非常理想的光动力活性 ${ }^{[7 \sim 10]}$. 为了寻 找具有长波吸收和结构稳定的光动力治疗药物, 进一步 考察和拓展叶绿素的化学反应范围, 从脱镁叶绿酸-a 甲 酯(1a)和脱镁叶绿酸-b 甲酯(1b) 开始, 在其二氢卟吩色 基上构建 $\beta, \beta$ 二㲵亚甲基和其他不饱和结构来扩展大环 分子的共轭体系, 研究了强吸电子基才的引入对四吡咯 大环的化学活性及其电子光谱所产生的影响, 进而完成 了一系列最大可见光吸收大于 $700 \mathrm{~nm}$ 的叶绿素类二氢 卟吩衍生物的合成.

首先将 $1 \mathrm{a}$ 在乙酸中回流转化成焦脱镁叶绿酸-a 甲 酯(1c), 不经分离, 直接在碱性条件下使其 E-环羰基与 丙二腈发生 Knoevenagel 缩合反应, 以 $41 \%$ 的产率生成 $13^{1}$-位二氧亚甲基取代的二氢卟吩(2). 选择四氧化锇和 高碘酸钠为氧化剂, 对 2 的 3-位乙烯基实施氧化, 分别 分离出 $13^{1}$-二氰亚甲基二氢卟吩醛 $(3,31 \%)$ 和焦脱镁叶 绿酸-d 甲酯 $(4,17 \%)$. 改用较弱氧化性的硝酸铊进行氧 化反应，结果得到 71\%的 C(3)-乙烯基的氧化产物 $\mathbf{5}$, 而 其 $13^{2}$-位的双键则保持不变. 二氢卟吩醛 $\mathbf{3}$ 与甲基溴化 镁的 Gerignad 反应以中等产率生成二氢卟吩醇 $(6,56 \%)$, 再选用 $N$-甲基吗啉氮氧化物和高钓酸四正丙基铵
(TPAP)对其进行氧化，以 43\%的产率得到 3a-乙酰基取 代的焦脱镁叶绿酸-a 甲酯(7). 考虑到不同位置引入吸 电子取代基团对大环分子的光物理性质可能产生不同 的影响, 利用脱镁叶绿酸-b 甲酯 $1 \mathbf{b}$ 的 7-位上原有甲酰 基与丙二腈进行缩合反应，在相同的 Knoevenagel 反应 条件下，仅仅得到 $9 \%$ 的期待产物-7-二氧亚甲基脱镁叶 绿酸-a 甲酯 $(8)$, 令人诧异的是分离出大量的起始原料 1a $(62 \%)$ (Scheme 1).

为了在 $\mathrm{N}^{21}-\mathrm{N}^{23}$ 轴向上扩展二氢卟吩的共轭体系, 通过二氢卟吩醛 3 与茮基三苯基磷的 Wittig 反应得到由 顺反异构体 $9 \mathrm{a}$ 和 $9 \mathrm{~b}$ 组成的混合物，二者没有进一步的 分离, 在三乙胺存在下与丙二睛直接进行 Knoevenagel 缩合反应，从反应混合物中分别出 $11 \%$ 的顺式 C(3b)-苯 基取代的二氢卟吩 $\mathbf{1 0 a}$ 和 34\% 的反式 C(3b)-苯基取代的 二氢卟吩 $\mathbf{1 0 b .}$

叶绿素降解产物中 20-位取代基团可以改善大环分 子的电子光谱 ${ }^{[8]}$, 为此, 在吡啶中将主产物 $E$-式异构体 $10 \mathrm{~b}$ 与乙酸镍共回流得到二氢卟吩镍配合物 $(11,61 \%)$, 然后在酸性条件下与 $N, N$-二甲基丙烯酰胺进行 Vilsmeier 反应，在 20-meso-位上引进甲酰乙烯基，以
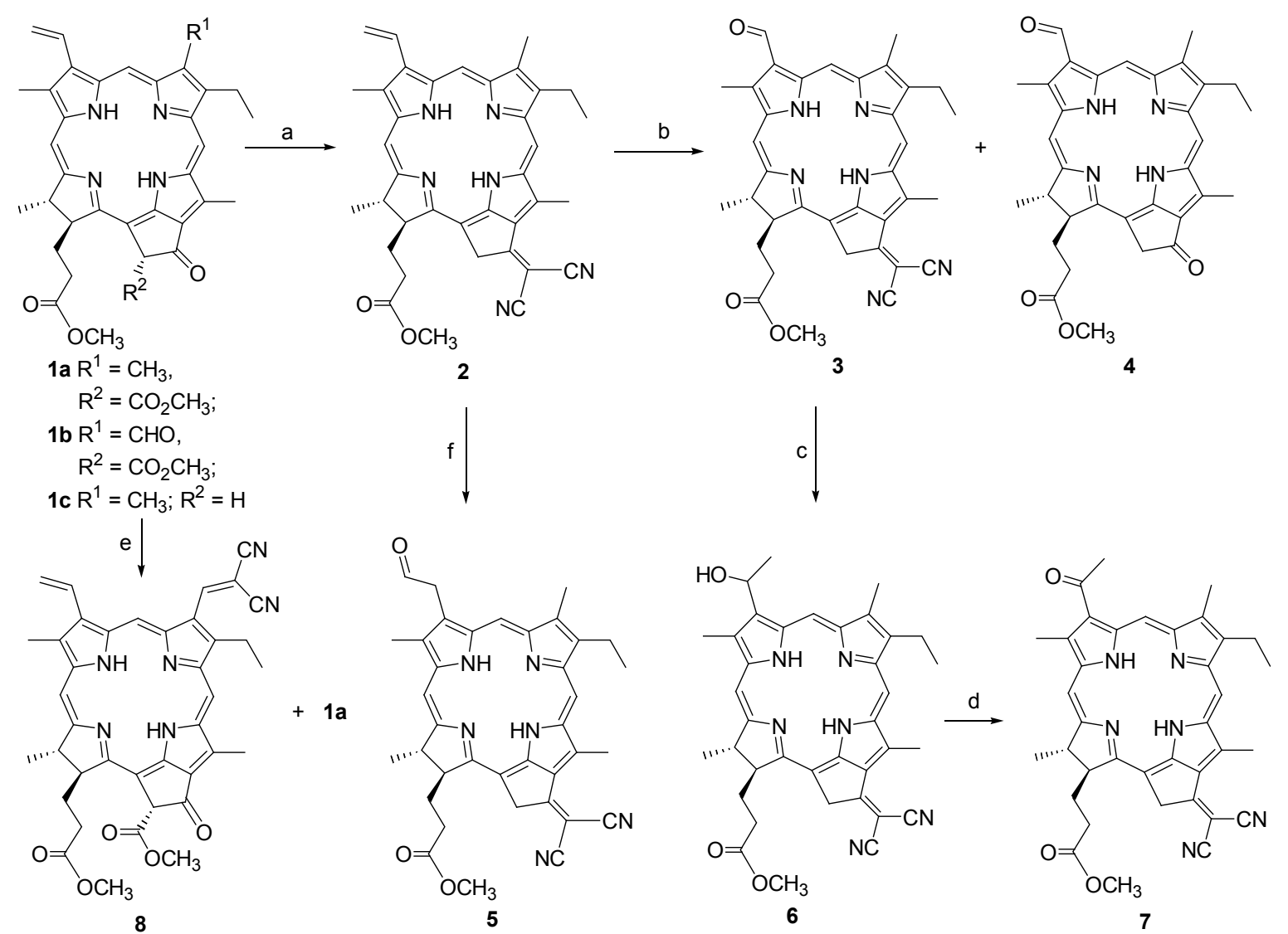

Reagents and conditions: (a) $\mathrm{AcOH} / \mathrm{CH}_{2}(\mathrm{CN})_{2} / \mathrm{NEt}_{3}$; (b) $\mathrm{OsO}_{4} / \mathrm{NalO}_{4} / \mathrm{Pyr}$; (c) $\mathrm{CH}_{3} \mathrm{MgBr} / \mathrm{THF}$; (d) $\mathrm{Pr}_{4} \mathrm{NRuO}_{4} / \mathrm{NMe}$ morpholine $\mathrm{N}$-oxide; (e) $\mathrm{CH}_{2}(\mathrm{CN})_{2} / \mathrm{NEt}_{3} ;$ (f) $\mathrm{TI}\left(\mathrm{NO}_{3}\right)_{3} / \mathrm{Na}_{2} \mathrm{SO}_{3}$

Scheme 1 
$24 \%$ 的产率分离出二氢卟吩醛镍配合物(12). 选择 $13^{2}$ 氧代焦脱镁叶绿酸-a 甲酯(13)与丙二睛进行缩合, 结果 $13^{1}$-位羰基转化成二氧亚甲基. 所生成的二氢卟吩酮 14 与 $N$-氯代丁二酰亚胺(NCS)进行亲电取代反应，以 $41 \%$ 的产率分离出 20-位氯代二氢卟吩 $\mathbf{1 5}$ (Scheme 2).

\section{1 结果与讨论}

\section{$1.113^{1}$-位 $\beta, \beta$-二氭亚甲基的引入对大环分子化学活 性的影响}

焦脱镁叶绿酸-a 甲酯(1c)的二氮杂[18]轮烯色基属 于芳香性共轭体系, 与其他芳香性 $\pi$-体系相似, 在其大 环分子的平面上可以形成相应的环电流，同时也表现出 极为明显的亲电取代的反应特征 ${ }^{[9]}$. 当二氢卟吩 2 的 $\mathrm{E}-$ 环羰基转换成 $\beta, \beta$-二氰亚甲基以后，在 $13^{1}$-位上引进一 个更为强烈的吸电子取代基团，其综合的电子效应导致 了芳香性大环上的环电流密度有所离散, 在一定程度上 钝化了二氢卟吩大环的化学反应活性. 与脱镁叶绿酸-a 甲酯 $1 \mathbf{a}$ 及其同系物相比，其亲电性的 Vilsmeier 反应、 氯代反应和氧化反应的速度以及反应产率都要相对降 低 $^{[6 \mathrm{c}, 11]}$.

$13^{1}$-位上的两个㲵基的吸电子作用提升了二氢卟吩
2 的氧化还原电位 ${ }^{[13]}$, 因而降低了大环分子中具有烯烃 特征的碳碳双键的还原能力. 焦脱镁叶绿酸-a 甲酯(1c) 与四氧化锇氧化反应仅需 $1 \mathrm{~h}$ ，且产率达到 $92 \%$; 而化合 物 2 的相同反应时间大约是前者的 3 倍，但 C(3)-双键的 氧化总产率却低于 70\%. 化合物 2 的 3-位乙烯基和 $13^{1}$ 位二氰亚甲基同时氧化后给出 $\mathbf{4}$, 而两个双键的氧化顺 序也是首先发生在电子云密度较强的 C(3)-乙烯基上, 即氧化途径 $\mathbf{a}$ 优先于途径 $\mathbf{b}$, 否则应该检测出焦脱镁叶 绿酸-a 甲酯(1c)的存在. 选用氧化能力较弱的硝酸铊作 为氧化剂对化合物 2 实施氧化, 只有 3-位乙烯基被氧化 成甲酰甲基, 而 $13^{2}$-位上具有较低电子云密度的碳碳双 键并没有发生任何变化.

当脱镁叶绿酸-b 甲酯 $\mathbf{1 b}$ 的 7-位甲酰基转化为二氰 亚甲基的时候，主要产物为 $\mathrm{C}(7)$-醛基的还原产物 1a, 而期待的缩合产物 7 的产率仅为 9\%. 由于 7-位甲酰基 所连接的双键游离于芳香性二氮杂 [18]轮烯之外，因而 带有烯醛结构的特性. 在碱性试剂促进下首先异构成烯 醇氧负离子 $\mathbf{B}$, 并于丙二睛发生取代反应形成烯醇醚 $\mathbf{C}$, 经电子转移转化成内盐中间体 $\mathbf{D}$, 所释出的极为活泼的 氰基甲醛 $\mathbf{E}$ 随即与三乙胺发生亲核加成反应而生成四 面体中间体 $\mathbf{F}$ ，在其羰基恢复过程中给出的负氢离子并

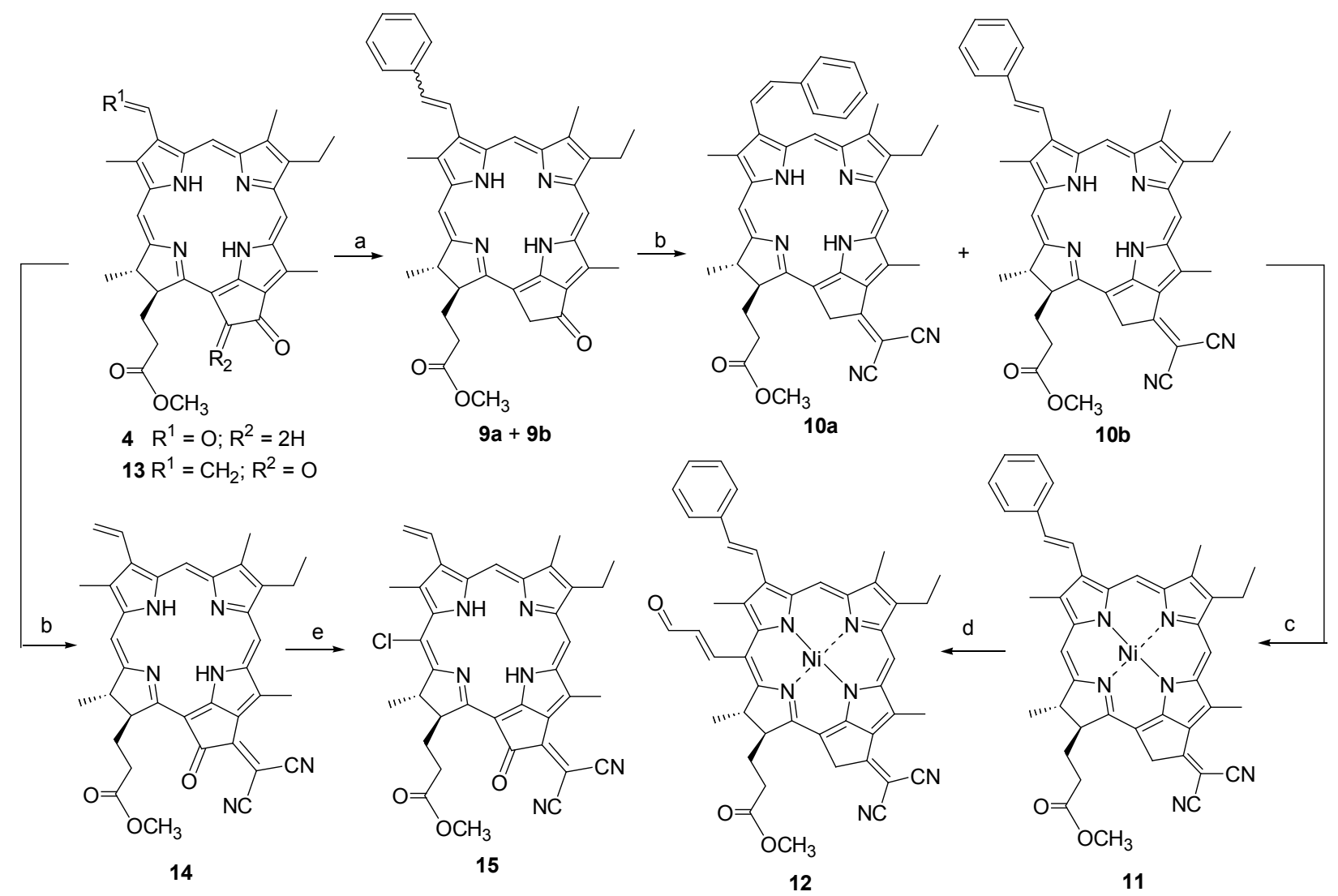

Reagents and conditions: (a) $\mathrm{PhCH}_{2} \mathrm{PPh}_{3} / \mathrm{NaOH} / \mathrm{THF}$; (b) $\mathrm{CH}_{2}(\mathrm{CN})_{2} / \mathrm{NEt}_{3} ;$ (c) $\mathrm{Ni}(\mathrm{OAc})_{2} / \mathrm{Pyr}$; (d) $\mathrm{Me}_{2} \mathrm{NCH}=\mathrm{CHCHO} / \mathrm{POCl}_{3} ;(\mathrm{e}) \mathrm{NCS} / \mathrm{CH}_{2} \mathrm{Cl}_{2}$

Scheme 2 

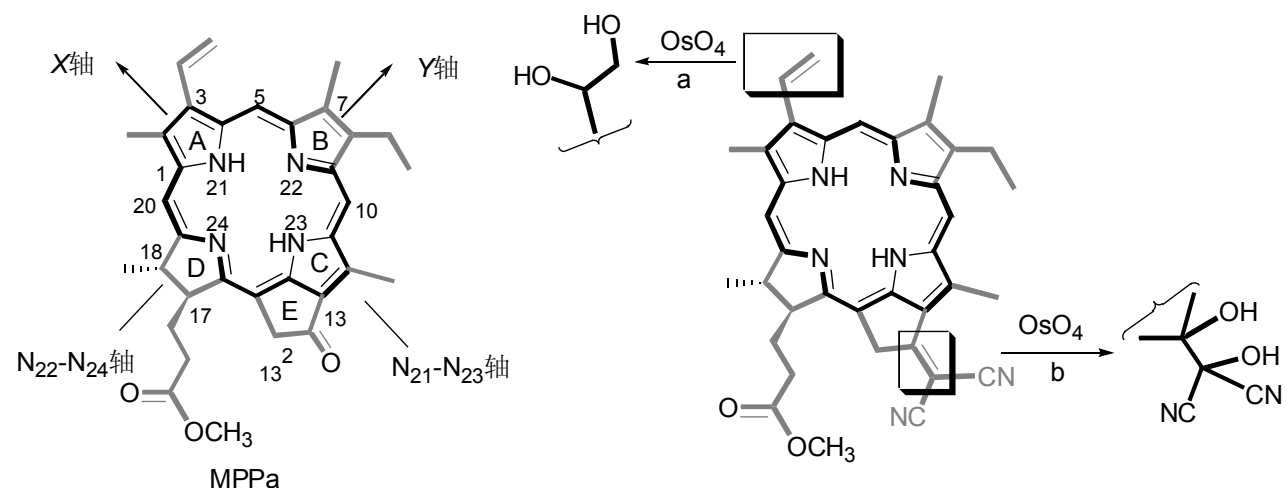

图 1 焦脱镁叶绿酸-a 甲酯的共轭体系及其 $13^{2}$-二氧亚甲基衍生物的氧化反应活性

Figure 1 Conjugated system of methyl pyropheophorbide-a and the oxidation reaction activity of its $13^{2}$-dicyanomethylene derivative

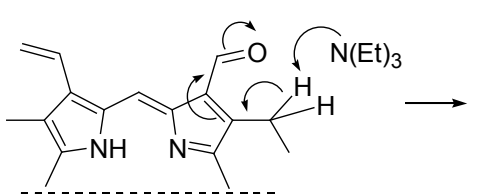

$1 \mathrm{~b}$

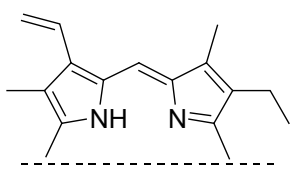

$1 \mathbf{a}$

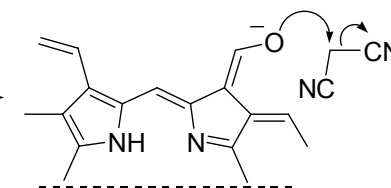

B
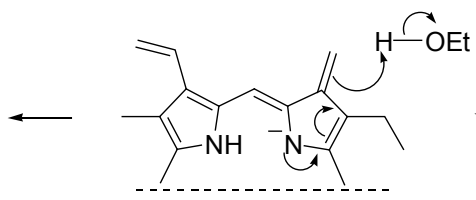

G<smiles>C#[N+]</smiles>

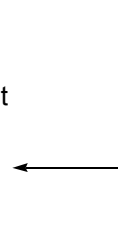

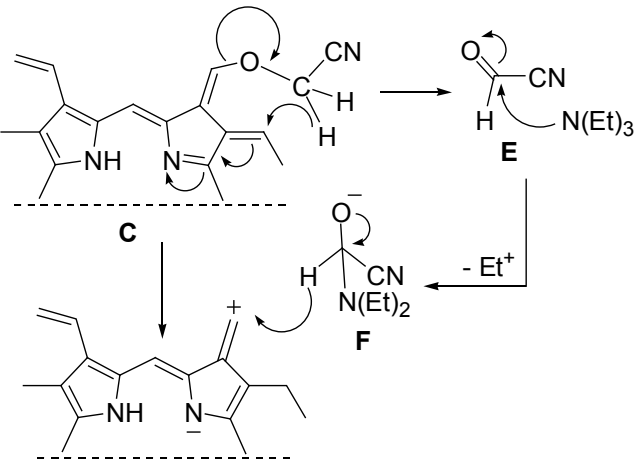

D

图 2 焦脱镁叶绿酸-b 甲酯向焦脱镁叶绿酸-a 甲酯的转化历程

Figure 2 Conversion process from methyl pyropheophorbide-b to methyl pyropheophorbide-a

与 $\mathbf{D}$ 的 $7 \mathrm{~b}$-位的正电荷连接, 最后, 负氮离子 $\mathbf{G}$ 夺取溶 剂中的质子而转化成脱镁叶绿酸-a 甲酯 $\mathbf{1 a}$.

脱镁叶绿酸-b 甲酯 $1 \mathrm{~b}$ 的 7-位甲酰基连接于游离于 芳香性氮杂轮烯之外的 B 环上, 在一定程度上更具有烯 醛的化学特征. 我们发现, 在其他的碱性或者酸性环境 中, 其 7-位甲酰基也很容易转化成甲基而形成脱镁叶绿 酸-a 甲酯 1a, 而在相同条件下, 脱镁叶绿酸-d 甲酯 4 和 12-位甲酰基脱镁叶绿酸-a 甲酯中的醛基则稳定存在 ${ }^{[7 \mathrm{c}]}$. 这种结构变化说明: 叶绿素大环上的 7-位甲酰基的稳定 性要远低于其他直接连接在芳香性色基上甲酰基, 或 许, 这是天然产物中所含叶绿素-a 的含量高于叶绿素-b 数倍的原因之一.

\section{$1.2 \beta, \beta$-二氰亚甲基的引入与其最大可见光吸收的变} 化

叶绿素及其衍生物的 $\mathrm{N}^{21}-\mathrm{N}^{23}$ 轴向组成了大环分子 中最为广泛的离域区域, 而 $\mathrm{N}^{22}-\mathrm{N}^{24}$ 轴向的两个吡咯子 环均没有包含在芳香性氮杂轮烯之内, 即使在 B 或者 D 子环上连接复键结构也不能有效地扩大二氢卟吩色基 的共轭 $\pi$-体系, 甚至在 7-位上连有甲酰基的时候, 其最 大可见光的吸收波长发生了大幅度的蓝移 ${ }^{[14]}$ (图 3). 然 而, 当羰基双键转化成 $\beta, \beta$-二氧亚甲基时, 其最大可见
光吸收(Qy 值)均产生相应幅度的红移. 二氢卟吩 2 的 Qy 吸收比焦脱镁叶绿酸-a 甲酯(1c)的相应波长红移了 $26 \mathrm{~nm} ; \mathrm{C}(3 \mathrm{~b})$-苯基取代的顺反异构体焦脱镁叶绿酸-a 甲 酯 9a 和 9b 与丙二睛发生 Knoevenagel 反应以后, 其缩 合产物 $10 \mathrm{a}$ 和 $10 \mathrm{~b}$ 的最大可见光吸收峰分别红移了 36 和 $26 \mathrm{~nm}^{[15]}$. 其中, 顺式异构体 $\mathbf{1 0 a}$ 的红移距离相对更 远，其原因可能是二氰亚甲基的引入改善了 $\mathrm{N}^{21}-\mathrm{N}^{23}$ 轴 向 $\pi$-体系的共轭效果, $\mathrm{C}(3)$-位上所连有的碳碳双键和苯

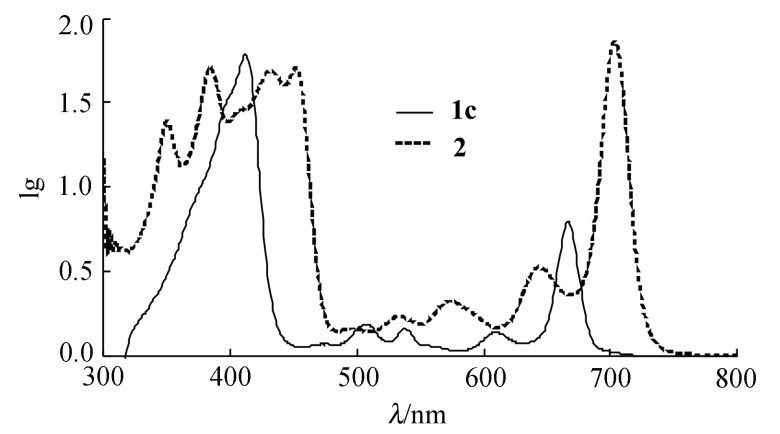

图 3 焦脱镁叶绿酸-a 甲酯(1c)和 $13^{2}$-二氧亚甲基衍生物 2 的 紫外-可见光谱

Figure 3 UV-Vis spectra of methyl pyropheophorbide-a (1c) and its $13^{2}$-dicyanomethylene derivative 
环与二氢卟吩色基共平面的程度则有所提高. 二氢卟吩 3, 6, 7 和 8 的 3-位上分别连有甲酰基、羟乙基、乙酰基 和甲酰乙基，与其 $13^{1}$-位非二氧亚甲基取代同系物的 Qy 值相比, 它们的 $\Delta Q y$ 值分别为 $32,27,30$ 和 $36 \mathrm{~nm}^{[14]}$. 当在 7-位引进二氧亚甲基时, 化合物 $\mathbf{8}$ 的最大可见光吸 收也比脱镁叶绿酸-b 甲酯 $\mathbf{1 b}$ 的相应吸收增长 $10 \mathrm{~nm}^{[15]}$.

与进行 Knoevenagel 反应的前体相比, $13^{1}$-二氧亚甲 基二氢卟吩 $\mathbf{2}$ 的电子光谱存在着两个明显的区别, 其一 是 Soret 谱带的半高全宽值(Fwhm 值)相对较大, 其二是 Soret 和 Qy 的峰值比(Soret/Qy)相对较小. 前者说明 $13^{1}$ 二氯亚甲基的引入一方面破坏了原有分子的对称性, 使 得原来相近的电子跃迁在能级上发生变化. 二氯亚甲基 本身也可能在 Soret 谱带附近形成了更多能量相近的 $\mathrm{n}-\pi$ 或者 $\pi-\pi^{*}$ 电子跃迁; 后者则由于 $\beta, \beta$-二氧亚甲基的 碳碳双键和碳氮三键扩展了大环分子的共轭区域, 相应 地降低了最高占有轨道(HOMO)和最低空轨道(LUMO) 之间的能级差别, 使得相应的电子跃迁需要相对较小的 能量激发. 同时, 最大的共轭 $\pi$-体系中的两个氧基沿着 $\mathrm{N}^{21}-\mathrm{N}^{23}$ 轴形成了一定的对称性, 也可能提高了 HOMO 轨道中的电子向 LUMO 轨道跃迁的发生几率.

在 $\beta, \beta$-二氧亚甲基取代的二氢卟吩光谱中, 普遍存 在着较低的 Soret 和 Qy 的峰值比, 因此, 其四吡咯大环 分子对最长吸收波长的光能够形成很强的吸收能力. 在 叶绿素大环上引进 $\beta, \beta$-二氧亚甲基结构, 既可以延伸分 子的最大可见光的吸收波长, 又可以提升相应的吸收强 度, 在合成新型 PDT 光敏剂的研究中更具有实际意义.

\section{2 结论}

通过对叶绿素降解产物的化学修饰, 在其二氢卟吩 周环上建立多种羰基结构, 利用其与丙二腈的 Knoevenagel 缩合反应, 在大环分子的不同位置上可以 建立 $\beta, \beta$-二氧亚甲基成分. 二氧亚甲基的引入不仅使得 最大可见光的吸收波长发生大幅度红移, 也能够有效地 提高 Qy 谱带的摩尔吸光系数, 同时, 对大环分子的反 应活性也产生明显的影响. 在叶绿素衍生物的碳架上建 立 $\beta, \beta$-二氧亚甲基结构, 可以调整和改善四吡咯大环分 子的光物理性质和化学反应活性, 为合成具有长波吸收 的新型叶绿素类二氢卟吩衍生物提供了新的切入途径.

\section{3 实验部分}

\section{1 仪器与试剂}

元素分析用 Perkin-Elmer 2400 型元素分析仪测定; IR 用 Perkin-Elmer 1730 型红外分光光度仪测定 $(\mathrm{KBr}$ 压 片); UV-vis 用 UV-160A 型紫外分光光度计测定; ${ }^{1} \mathrm{H}$ NMR 用 Beucker ARX-400 型核磁共振仪测定, 内标为
TMS; 所用试剂均为分析纯和化学纯. 脱镁叶绿酸-a 甲 酯 1a 按文献[13]制备; 脱镁叶绿酸-b 甲酯(9)按文献[15] 制备; $13^{2}$-氧代焦脱镁叶绿酸-a 甲酯 13 按文献[17]制备; 二氢卟吩 2,3 和 4 按文献 $[18,19]$ 制备.

\subsection{3 -甲酰甲基- $13^{1}-\beta, \beta$-二氭亚甲基-3-去乙烯基焦脱 镁叶绿酸 $-d$ 甲酯(5)的合成}

在 $50 \mathrm{~mL}$ 二氯甲烷和 $25 \mathrm{~mL}$ 甲醇混合液中溶解 200 $\mathrm{mg}$ 的化合物 $2(0.335 \mathrm{mmol})$, 于 $0{ }^{\circ} \mathrm{C}$ 下将溶解于 $30 \mathrm{~mL}$ 甲醇的 $150 \mathrm{mg}$ 三水硝酸铊迅速加入到反应体系中, 并 在此温度下摚拌反应 $50 \mathrm{~min}$. 然后加入 $30 \mathrm{~mL}$ 亚硫酸钠 的甲醇饱和溶液, 室温下摚拌 $30 \mathrm{~min}$ 后, 滴加 $2 \mathrm{~mL}$ 浓 盐酸溶液, 将所生成的白色沉淀过滤除去. 加入 $30 \mathrm{~mL}$ 水和 $40 \mathrm{~mL}$ 二氯甲烷, 分出有机层, 用无水硫酸钠干燥 后除去溶剂, 将所得暗红色固体重新溶解于 $10 \mathrm{~mL} 80 \%$ 的甲酸溶液中, 室温摚拌 $4 \mathrm{~h}$, 再加入 $100 \mathrm{~mL}$ 水和 25 $\mathrm{mL}$ 二氯甲烷，分出有机层后，用二氯甲烷萃取 (30 $\mathrm{mL} \times 2)$, 合并有机相, 水洗后干燥, 浓缩, 剩余物经硅 胶柱层析分离[洗脱剂: $V$ (石油醚) $: V($ 乙酸乙酯 $)=2$ : 1], 得 $146 \mathrm{mg}$ 暗红色固体产物 5 (0.238 mmol), 产率 71\%. m.p. $188 \sim 191{ }^{\circ} \mathrm{C}$; UV-Vis $\left(\mathrm{CHCl}_{3}\right) \lambda_{\max }$ (relative intensity): 430, 0.95, 449 (1.00), 529 (0.13), 569 (0.16), 640 (0.29), $698(1.08) \mathrm{nm} ;{ }^{1} \mathrm{H}$ NMR (400 MHz, $\left.\mathrm{CDCl}_{3}\right) \delta$ : -1.11 (br, $1 \mathrm{H}, \mathrm{NH}), 0.65$ (br s, 1H, NH), 1.56 (t, $J=7.6$ $\left.\mathrm{Hz}, 3 \mathrm{H}, 8-\mathrm{CH}_{3}\right), 1.79$ (d, $\left.J=7.3 \mathrm{~Hz}, 3 \mathrm{H}, 18-\mathrm{CH}_{3}\right), 1.98 \sim$ $2.08,2.23 \sim 2.35,2.42 \sim 2.60$ (each $\mathrm{m}$, all $4 \mathrm{H}, 17 \mathrm{a}+7 \mathrm{~b}-\mathrm{H}$ ), 2.97, 3.19, 3.28, 3.66 (each s, each $3 \mathrm{H}, \mathrm{CH}_{3}+\mathrm{OCH}_{3}$ ), 3.57 (q, $J=7.6 \mathrm{~Hz}, 2 \mathrm{H}, 8 \mathrm{a}-\mathrm{H}), 4.05(\mathrm{~d}, J=9.5 \mathrm{~Hz}, 1 \mathrm{H}, 17-\mathrm{H})$, 4.34 (q, $J=7.3 \mathrm{~Hz}, 1 \mathrm{H}, 18-\mathrm{H}), 4.83(\mathrm{~d}, J=1.5 \mathrm{~Hz}, 2 \mathrm{H}$, 3a-H), 8.41, 8.85, 8.95 (each s, each $1 \mathrm{H}$, meso-H), 10.15 (d, $J=1.5 \mathrm{~Hz}, 1 \mathrm{H}$, formyl-H); IR $(\mathrm{KBr}) v: 3443(\mathrm{~N}-\mathrm{H})$, $2958(\mathrm{C}-\mathrm{H}), 1689 \sim 1737(\mathrm{C}=\mathrm{O}), 1655(\mathrm{C}=\mathrm{C}), 1527$ (chlorin skeleton), 1400, 1286, 1189, $1093 \mathrm{~cm}^{-1}$. Anal. calcd for $\mathrm{C}_{37} \mathrm{H}_{36} \mathrm{~N}_{6} \mathrm{O}_{3}$ : C 72.53, H 5.92, N 13.72; found $\mathrm{C}$ 72.38, H 5.77, N 13.68 .

\subsection{3 -(1-羟基乙基)-13 ${ }^{1}$ - $\beta, \beta$-二氰亚甲基-3-去乙烯基 焦脱镁叶绿酸-a 甲酯(6)的合成}

将 $260 \mathrm{mg}$ 化合物 $3(0.434 \mathrm{mmol})$ 溶解于 $20 \mathrm{~mL}$ 干 燥的四氢呋喃中, 在氮气保护, $0{ }^{\circ} \mathrm{C}$ 搅拌下缓慢滴加 1 $\mathrm{mL}$ 的 $1 \mathrm{~mol} / \mathrm{L}$ 甲基溴化镁乙醚溶液，在相同条件下再继 续搅拌 $30 \mathrm{~min}$, 向反应体系加入 $25 \mathrm{~mL}$ 的饱和氯化铵溶 液, 搅拌 $15 \mathrm{~min}$, 后再加入 $20 \mathrm{~mL}$ 的二氯甲烷, 分出有机 层, 用二氯甲烷萃取水相 $(15 \mathrm{~mL} \times 2)$, 合并有机相并浓 缩, 将剩余物经柱层析分离 [洗脱剂: $V$ ( 乙酸乙 酯)：V(正己醇 $)=1 ： 2$ ]得 $149 \mathrm{mg}$ 黑绿色固体 $6(0.243$ $\mathrm{mmol})$, 产率为 $56 \%$. m.p. $212 \sim 215{ }^{\circ} \mathrm{C}$; UV-Vis $\left(\mathrm{CHCl}_{3}\right)$ 
$\lambda_{\max }$ (relative intensity): 382 (0.91), 431 (0.92), 449 (0.95), 532 (0.19), 572 (0.22), 641 (0.32), 698 (1.00) nm; ${ }^{1} \mathrm{H}$ NMR $\left(\mathrm{CDCl}_{3}\right) \delta:-1.25(-1.29)$ (brs, $\left.1 \mathrm{H}, \mathrm{NH}\right), 0.65(0.70)$ (brs, 1H, NH), 1.60 (t, $J=7.5 \mathrm{~Hz}, 3 \mathrm{H}, 8 \mathrm{~b}-\mathrm{H}), 1.75$ (1.73) (d, $J=7.2 \mathrm{~Hz}, 3 \mathrm{H}, 18-\mathrm{CH}_{3}$ ), 2.04 2.28, 2.32 2.60 (each m, all $4 \mathrm{H}, 17 \mathrm{a}+17 \mathrm{~b}-\mathrm{H}), 2.13(2.11)(\mathrm{d}, J=6.6 \mathrm{~Hz}, 3 \mathrm{H}$, 3a- $\mathrm{CH}_{3}$ ), 2.86, 2.93, 3.35 (3.21), 3.64 (3.62) (each s, each $\left.3 \mathrm{H}, 12 \mathrm{H}, \mathrm{CH}_{3}+\mathrm{OCH}_{3}\right), 3.60$ (q, $\left.J=7.5 \mathrm{~Hz}, 2 \mathrm{H}, 8 \mathrm{a}-\mathrm{H}\right)$, $3.87 \sim 3.90(\mathrm{~m}, 1 \mathrm{H}, 18-\mathrm{H}), 4.25 \sim 4.37(\mathrm{~m}, 1 \mathrm{H}, 18-\mathrm{H}), 4.94$ (4.88) (d, $\left.J=19.7 \mathrm{~Hz}, 1 \mathrm{H}, 13^{2}-\mathrm{H}\right), 5.00$ (5.49) (d, $J=19.7$ $\left.\mathrm{Hz}, 1 \mathrm{H}, 13^{2}-\mathrm{H}\right), 6.36$ (6.34) (t, $\left.J=6.6 \mathrm{~Hz}, 1 \mathrm{H}, 3 \mathrm{a}-\mathrm{H}\right), 8.33$ (8.32), 8.88 (8.84), 9.58 (9.54) (each s, each $1 \mathrm{H}, 3 \mathrm{H}$, meso-H); IR (KBr) v: $3445(\mathrm{~N}-\mathrm{H}), 3168(\mathrm{O}-\mathrm{H}), 2954$ $(\mathrm{C}-\mathrm{H}), 1688 \sim 1728(\mathrm{C}=\mathrm{O}), 1654(\mathrm{C}=\mathrm{C}), 1560$ (chlorin skeleton), 1541, 1400, $1084 \mathrm{~cm}^{-1}$. Anal. calcd for $\mathrm{C}_{37} \mathrm{H}_{38} \mathrm{~N}_{6} \mathrm{O}_{3}$ : C 72.29, H 6.23, N 13.67; found C 72.49, H 6.49, N 13.82 .

\subsection{3 -乙酰基- $13^{1}-\beta, \beta$-二氭亚甲基-3-去乙烯基焦脱镁} 叶绿酸- $\mathrm{a}$ 甲酯(7)的合成

在 $25 \mathrm{~mL}$ 的干燥二氯甲烷中溶解 $139 \mathrm{mg}$ 二氢卟吩 醇 $6(0.226 \mathrm{mmol})$, 在氮气保护下, 迅速加入 $20 \mathrm{mg} \mathrm{N}$ 甲基吗啉氧化物并避光摚拌 $15 \mathrm{~min}$, 再小量分批加入 30 $\mathrm{mg}$ 高钓酸四丙基铵(TPAP), 用薄板层析监测反应, 摚 拌 $1.5 \mathrm{~h}$ 后反应结束, 加入 $20 \mathrm{~mL}$ 水, 静置过夜, 分出水 层后用二氯甲烷萃取 $(15 \mathrm{~mL} \times 3)$, 合并有机层, 用无水 硫酸钠干燥, 除去溶剂, 所得剩余物经柱层析分离 [洗 脱剂: $V$ (乙酸乙酯) $: V$ (正己烷) $=1: 4$ ]得 $60 \mathrm{mg}$ 黑色固 体 7 (0.097 mmol), 产率 43\%. m.p. 198 201 ${ }^{\circ} \mathrm{C}$; UV-Vis $\left(\mathrm{CHCl}_{3}\right) \lambda_{\max }$ (relative intensity): 379 (0.70), 423 (1.00), 585 (0.27), 659 (0.24), 715 (0.44) nm; ${ }^{1} \mathrm{H}$ NMR (400 MHz, $\left.\mathrm{CDCl}_{3}\right) \delta:-1.59($ br s, $1 \mathrm{H}, \mathrm{NH}), 0.61$ (br s, $\left.1 \mathrm{H}, \mathrm{NH}\right)$, 1.64 (t, $\left.J=7.6 \mathrm{~Hz}, 3 \mathrm{H}, 8-\mathrm{CH}_{3}\right), 1.78(\mathrm{~d}, J=7.3 \mathrm{~Hz}, 3 \mathrm{H}$, $18-\mathrm{CH}_{3}$ ), $2.23 \sim 2.35,2.50 \sim 2.60,2.62 \sim 2.72$ (each m, all $4 \mathrm{H}, 17 \mathrm{a}+17 \mathrm{~b}-\mathrm{H}$ ), 3.16, 3.26, 3.30, 3.60, 3.61 (each s, each $3 \mathrm{H}, \mathrm{CH}_{3}+\mathrm{OCH}_{3}$ ), 3.60 (q, $\left.J=7.6 \mathrm{~Hz}, 2 \mathrm{H}, 8 \mathrm{a}-\mathrm{H}\right), 4.23$ (dd, $J=6.0,2.4 \mathrm{~Hz}, 1 \mathrm{H}, 17-\mathrm{H}), 4.42(\mathrm{~d}, J=7.3 \mathrm{~Hz}, 1 \mathrm{H}, 18-\mathrm{H})$, $5.05\left(\mathrm{~d}, J=19.7 \mathrm{~Hz}, 1 \mathrm{H}, 13^{2}-\mathrm{H}\right), 5.16(\mathrm{~d}, J=19.7 \mathrm{~Hz}, 1 \mathrm{H}$, $\left.13^{2}-\mathrm{H}\right), 8.39,9.05,9.36$ (each s, each $1 \mathrm{H}$, meso-H); IR (KBr) v: $3342(\mathrm{~N}-\mathrm{H}), 2915,2847(\mathrm{C}-\mathrm{H}), 1739 \sim 1692$ $(\mathrm{C}=\mathrm{O}), 1608(\mathrm{C}=\mathrm{C}), 1498$ (chlorin skeleton), 1437, 1365, 1199, 1123, $982 \mathrm{~cm}^{-1}$. Anal. calcd for $\mathrm{C}_{37} \mathrm{H}_{36} \mathrm{~N}_{6} \mathrm{O}_{3}$ : C 72.53, H 5.92, N 13.72; found C 72.40, H 6.06, N 13.61.

\section{$3.57-\beta, \beta$-二氭亚甲基脱镁叶绿酸-a 甲酯(8)和脱镁叶} 绿酸- $a$ 甲酯(1a)的合成

将 $300 \mathrm{mg}$ 脱镁叶绿酸-b 甲酯 $\mathbf{1 b}(0.483 \mathrm{mmol})$ 溶解
于 $40 \mathrm{~mL}$ 无水乙醇中, 加入 $367 \mathrm{mg}(5.550 \mathrm{mmol})$ 丙二腈, 再加入 $0.5 \mathrm{~mL}$ 三乙胺, 氮气保护下室温反应 $3 \mathrm{~h}$, 加入 水和二氯甲烷分层, 分出有机层并水洗, 水层用二氯甲 烷萃取 $(100 \mathrm{~mL} \times 3)$, 合并有机相后再用 $1 \%$ 盐酸水溶液 和冷水洗涤, 有机相干燥后减压浓缩, 硅胶柱层析分离 [展开剂: $V$ (乙酸乙酯) $: V$ (正己醇) $=3: 1$ ], 得 $29 \mathrm{mg}$ 暗 黄色的固体 $8(0.044 \mathrm{mmol}, 9 \%)$ 和 $182 \mathrm{mg}$ 黑绿色固体 1a (0.300 mmol, 62\%).

8: m.p. $215 \sim 217{ }^{\circ} \mathrm{C}$; UV-Vis $\left(\mathrm{CHCl}_{3}\right) \lambda_{\max }$ (relative intensity): 404 (1.00), 502 (0.11), $532(0.10), 606(0.08)$, $666(0.32) \mathrm{nm} ;{ }^{1} \mathrm{H}$ NMR $\left(400 \mathrm{MHz}, \mathrm{CDCl}_{3}\right) \delta:-1.76(\mathrm{br}$ s, $1 \mathrm{H}, \mathrm{NH}),-1.07(\mathrm{br} \mathrm{s}, 1 \mathrm{H}, \mathrm{NH}), 1.73(\mathrm{t}, J=7.6 \mathrm{~Hz}, 3 \mathrm{H}$, $\left.8-\mathrm{CH}_{3}\right), 1.81\left(\mathrm{~d}, J=7.1 \mathrm{~Hz}, 3 \mathrm{H}, 18-\mathrm{CH}_{3}\right), 1.95 \sim 2.04$, $2.28 \sim 2.38,2.41 \sim 2.58,2.60 \sim 2.71$ (each $\mathrm{m}$, all $4 \mathrm{H}$, $17 \mathrm{a}+17 \mathrm{~b}-\mathrm{H}$ ), 3.28, 3.45, 3.72, 3.90 (each s, each $3 \mathrm{H}$, $\left.\mathrm{CH}_{3}+\mathrm{OCH}_{3}\right), 4.30(\mathrm{q}, J=7.3 \mathrm{~Hz}, 1 \mathrm{H}, 18-\mathrm{H}), 4.45$ (q, $J=$ $7.6 \mathrm{~Hz}, 2 \mathrm{H}, 8 \mathrm{a}-\mathrm{H}), 4.90$ (d, $J=8.0 \mathrm{~Hz}, 1 \mathrm{H}, 17-\mathrm{H}), 6.16$ (d, $J=12.0 \mathrm{~Hz}, 1 \mathrm{H}, 3 \mathrm{~b}-\mathrm{H}), 6.32\left(\mathrm{~s}, 1 \mathrm{H}, 13^{2}-\mathrm{H}\right), 6.34$ (d, $J=$ $16.0 \mathrm{~Hz}, 1 \mathrm{H}, 3 \mathrm{~b}-\mathrm{H}), 7.51$ (s, $1 \mathrm{H}, 7 \mathrm{a}-\mathrm{H}), 8.03$ (dd, $J=16.0$, $12.0 \mathrm{~Hz}, 1 \mathrm{H}, 3 \mathrm{a}-\mathrm{H}$ ), 8.56, 8.75, 9.73 (each s, each $1 \mathrm{H}$, meso-H); IR (KBr) v: $3446(\mathrm{~N}-\mathrm{H}), 2926(\mathrm{C}-\mathrm{H}), 1740 \sim$ $1680(\mathrm{C}=\mathrm{O}), 1612(\mathrm{C}=\mathrm{C}), 1530$ (chlorin skeleton), 1461, $1307,1131,1072,1000 \mathrm{~cm}^{-1}$. Anal. calcd for $\mathrm{C}_{39} \mathrm{H}_{36} \mathrm{~N}_{6} \mathrm{O}_{5}$ : C 70.04, H 5.43, N 12.57; found C 69.91, H 5.26, N 12.61 . $1 \mathbf{a}$ 的分析数据与文献 $[16]$ 一致

3.6 (Z)-3b-苯基-13 $1{ }^{1}-\beta, \beta$-二氭亚甲基- $13^{1}$-去氧焦脱镁 叶绿酸-b 甲酯(10a)和 $(E)-3 b$ - 苯基- $13^{1}-\beta, \beta$-二氭亚甲 基-13 ${ }^{1}$-去氧焦脱镁叶绿酸-甲酯(10b)的合成

将 $411 \mathrm{mg}$ (0.746 mmol) 4 和 $411 \mathrm{mg}$ 氯化苠基三苯 基膦(1.054 mmol)溶解于 $120 \mathrm{~mL}$ 二氯甲烷中, 然后搅拌 下加入溶解于 $24 \mathrm{~mL}$ 水的 $80 \mathrm{mg}$ 氢氧化钠, 室温下氮气 保护搅拌 $30 \mathrm{~min}$, 再加入 $40 \mathrm{~mL}$ 冰水和 $40 \mathrm{~mL}$ 二氯甲烷, 用 $2 \%$ 的盐酸将反应体系调至 $\mathrm{pH}$ 为 3 , 分出有机层, 水 层用二氯甲烷萃取两次, 合并有机相, 用无水硫酸钠干 燥, 除去溶剂后经柱层析分离[洗脱液： $V$ (石油醚)： $V$ (乙酸乙酯 $)=3: 1$ ] 分别得到 $354 \mathrm{mg}$ 由 $9 \mathbf{a}$ 和 $9 \mathbf{b}$ 组成的 橙色混合物. 将所得固体溶解于 $40 \mathrm{~mL}$ 无水乙醇中, 再 加入 $725 \mathrm{mg}$ 丙二腈 $(10.975 \mathrm{mmol})$ 和 $0.5 \mathrm{~mL}$ 三乙胺, 氮 气保护下回流反应 $5 \mathrm{~h}$, 加入水和二氯甲烷分层, 分出 有机层并水洗，水层用二氯甲烷萃取 $(45 \mathrm{~mL} \times 3)$, 合并 有机相后再用 $1 \%$ 盐酸水溶液和冷水洗涤, 有机相干燥 后减压浓缩, 硅胶柱层析分离[展开剂: $V$ (乙酸乙酯) : $V($ 正己醇 $)=3: 1$ 分别得到 $55 \mathrm{mg}$ 橙色固体 10a $(0.082$ mmol, 11\%)和 $171 \mathrm{mg}$ 墨绿色固体 $10 \mathrm{~b}(0.254 \mathrm{mmol}$, $34 \%)$. 
10a: UV-Vis $\left(\mathrm{CHCl}_{3}\right) \lambda_{\max }$ (relative intensity): 386 (0.88), 434 (0.94), 452 (1.00), 495 (0.16), 533 (0.19), 574 (0.23), 644 (0.34), 703 (1.01) nm; ${ }^{1} \mathrm{H}$ NMR $\left(\mathrm{CDCl}_{3}\right) \delta$ : -0.86 (br s, $1 \mathrm{H}, \mathrm{NH}), 0.78$ (br s, $1 \mathrm{H}, \mathrm{NH}), 1.58$ (t, $J=7.8$ $\left.\mathrm{Hz}, 3 \mathrm{H}, 8 \mathrm{~b}-\mathrm{CH}_{3}\right), 1.75$ (d, $\left.J=7.2 \mathrm{~Hz}, 3 \mathrm{H}, 18-\mathrm{CH}_{3}\right), 2.20 \sim$ 2.35, 2.46 2.64 (each m, all 4H, 17a+17b-H), 2.90, 3.00, $3.53,3.64$ (each s, each $3 \mathrm{H}, \mathrm{CH}_{3}+\mathrm{OCH}_{3}$ ), 3.53 (q, $J=7.8$ $\mathrm{Hz}, 2 \mathrm{H}, 8 \mathrm{a}-\mathrm{H}$ ), 4.06 4.15, 4.18 4.35 (each m, each $1 \mathrm{H}$, $17+18-\mathrm{H}), 5.38\left(\mathrm{~d}, J=20 \mathrm{~Hz}, 1 \mathrm{H}, 13^{2}-\mathrm{H}\right), 5.52(\mathrm{~d}, J=20$ $\left.\mathrm{Hz}, 1 \mathrm{H}, 13^{2}-\mathrm{H}\right), 6.94 \sim 7.25(\mathrm{~m}, 3 \mathrm{H}, \mathrm{Ph}-\mathrm{H}), 7.35(\mathrm{~d}, J=$ $11.4 \mathrm{~Hz}, 1 \mathrm{~b}-\mathrm{H}), 7.52$ (m, 1H, Ph-H), 7.54 (d, $J=11.4 \mathrm{~Hz}$, 1H, 3a-H), 8.27, 9.02, 9.10 (each s, each 1H, meso-H); IR $(\mathrm{KBr}) v: 3448,3180(\mathrm{~N}-\mathrm{H}), 2929,2866(\mathrm{C}-\mathrm{H}), 1741 \sim$ $1689(\mathrm{C}=\mathrm{O}), 1638(\mathrm{C}=\mathrm{C}), 1528$ (chlorin skeleton), 1400, 1173, 1071, 1040, 968, $728 \mathrm{~cm}^{-1}$. Anal. calcd for $\mathrm{C}_{43} \mathrm{H}_{40} \mathrm{~N}_{6} \mathrm{O}_{2}$ : C 76.76, H 5.99, N 12.49; found C 76.60, H 5.81, N 12.24 .

10b: UV-Vis $\left(\mathrm{CHCl}_{3}\right) \lambda_{\max }$ (relative intensity): 390 (0.84), 434 (0.89), 455 (1.00), 496 (0.08), 577 (0.12), 649 (0.27), 710 (0.97) nm; ${ }^{1} \mathrm{H} \mathrm{NMR}\left(\mathrm{CDCl}_{3}\right) \delta$ : -0.98 (br s, $1 \mathrm{H}, \mathrm{NH}), 0.06$ (br s, $1 \mathrm{H}, \mathrm{NH}), 1.61$ (t, $J=7.8 \mathrm{~Hz}, 3 \mathrm{H}$, $\left.8 \mathrm{~b}-\mathrm{CH}_{3}\right), 1.75\left(\mathrm{~d}, J=7.2 \mathrm{~Hz}, 3 \mathrm{H}, 18-\mathrm{CH}_{3}\right), 2.17 \sim 2.36$, $2.40 \sim 2.63$ (each $\mathrm{m}$, all $4 \mathrm{H}, 17 \mathrm{a}+17 \mathrm{~b}-\mathrm{H}), 3.09,3.32,3.34$, 3.63 (each s, each $3 \mathrm{H}, \mathrm{CH}_{3}+\mathrm{OCH}_{3}$ ), 3.60 (q, $J=7.8 \mathrm{~Hz}$, $2 \mathrm{H}, 8 \mathrm{a}-\mathrm{H}$ ), $4.08 \sim 4.17,4.24 \sim 4.40$ (each m, each $1 \mathrm{H}, 17+$ $18-\mathrm{H}), 5.40$ (d, $\left.J=20 \mathrm{~Hz}, 1 \mathrm{H}, 13^{2}-\mathrm{H}\right), 5.54$ (d, $J=20 \mathrm{~Hz}$, $\left.1 \mathrm{H}, 13^{2}-\mathrm{H}\right), 7.44 \sim 7.57$ (m, 3H, Ph-H), 7.55 (d, $J=16.3$ $\mathrm{Hz}, 1 \mathrm{H}, 3 \mathrm{~b}-\mathrm{H}), 7.78$ (d, J=7.2 Hz, 1H, Ph-H), 8.13 (d, $J=$ $16.3 \mathrm{~Hz}, 1 \mathrm{H}, 3 \mathrm{a}-\mathrm{H}$ ), 8.36, 9.10, 9.12 (each s, each 1H, meso-H). IR (KBr) v: $3449(\mathrm{~N}-\mathrm{H}), 2924(\mathrm{C}-\mathrm{H}), 1736$ $(\mathrm{C}=\mathrm{O}), 1684(\mathrm{C}=\mathrm{C}), 1560$ (chlorin skeleton), 1459, 1341, 1086, 1040, 976, $669 \mathrm{~cm}^{-1}$. Anal. calcd for $\mathrm{C}_{43} \mathrm{H}_{40^{-}}$ $\mathrm{N}_{6} \mathrm{O}_{2}$ : C 76.76, H 5.99, N 12.49; found C 76.89, H 6.13, N 12.67 .

\section{7 (E)-3b-苯基-13 ${ }^{1}-\beta, \beta$-二氰亚甲基- $13^{1}$-去氧焦脱镁 叶绿酸-b 甲酯镍配合物(11)的合成}

将 $129 \mathrm{mg}$ 化合物 10a (0.192 mmol)溶解于 $30 \mathrm{~mL}$ 吡 啶中, 再向反应体系加入 $0.8 \mathrm{~g}$ 研细的醋酸镍, $90{ }^{\circ} \mathrm{C}$ 搅 拌反应 $4 \mathrm{~h}$, 减压除去反应溶剂, 将剩余物溶解于 $60 \mathrm{~mL}$ 二氯甲烷中, 再加入 $4 \mathrm{~mL}$ 乙酸和 $60 \mathrm{~mL}$ 水进行分层, 分 出有机相并水洗两次, 干燥后用重氮甲烷处理, 除去溶 剂后将剩余物通过柱层析分离[洗脱剂: $V$ (乙酸乙酯) : $V($ 正己烷 $)=1 ： 3$ 得 $85 \mathrm{mg}$ 亮绿色固体 $11(0.117 \mathrm{mmol})$, 产率 61\%. m.p. 207 $210{ }^{\circ} \mathrm{C}$; UV-Vis $\left(\mathrm{CHCl}_{3}\right) \lambda_{\max }$ (relative intensity): 396 (1.00), 422 (0.89), 519 (0.15), 698
(0.85) nm; ${ }^{1} \mathrm{H}$ NMR (400 MHz, $\left.\mathrm{CDCl}_{3}\right) \delta: 1.27$ (t, $J=7.2$ $\left.\mathrm{Hz}, 3 \mathrm{H}, 8-\mathrm{CH}_{3}\right), 1.56$ (d, J=7.1 Hz, 3H, 18- $\mathrm{CH}_{3}$ ), 1.90 2.20, 2.25 2.60 (each m, all 4H, 17a+17b-H), 3.06, 3.19, 3.46, 3.62 (each s, each $3 \mathrm{H}, \mathrm{CH}_{3}+\mathrm{OCH}_{3}$ ), 3.51 (q, $J=7.2$ $\mathrm{Hz}, 2 \mathrm{H}, 8 \mathrm{a}-\mathrm{H}), 3.98$ (d, $J=8.6 \mathrm{~Hz}, 1 \mathrm{H}, 17-\mathrm{H}), 4.28$ (q, $J=$ $7.1 \mathrm{~Hz}, 1 \mathrm{H}, 17-\mathrm{H}), 4.81$ (d, $\left.J=4.2 \mathrm{~Hz}, 2 \mathrm{H}, 13^{2}-\mathrm{H}\right), 7.28$ (t, $J=7.6 \mathrm{~Hz}, 1 \mathrm{H}, \mathrm{Ph}-\mathrm{H}), 7.48$ (t, $J=7.6 \mathrm{~Hz}, 2 \mathrm{H}, \mathrm{Ph}-\mathrm{H}), 7.40$ (d, $J=16.1 \mathrm{~Hz}, 1 \mathrm{H}, 3 \mathrm{~b}-\mathrm{H}), 7.76$ (d, $J=7.8 \mathrm{~Hz}, 2 \mathrm{H}, \mathrm{Ph}-\mathrm{H})$, 8.03 (d, $J=16.1 \mathrm{~Hz}, 1 \mathrm{H}, 3 \mathrm{a}-\mathrm{H}$ ), 8.17, 9.04, 9.25 (each s, each 1H, meso-H); IR (KBr) v: $3447(\mathrm{~N}-\mathrm{H}), 2960(\mathrm{C}-$ $\mathrm{H}), 1737 \sim 1680(\mathrm{C}=\mathrm{O}), 1654(\mathrm{C}=\mathrm{C}), 1541$ (chlorin skeleton), 1521, 1458, 1397, 1174, 1068, $1050 \mathrm{~cm}^{-1}$. Anal. calcd for $\mathrm{C}_{43} \mathrm{H}_{38} \mathrm{~N}_{6} \mathrm{NiO}_{2}$ : C 70.80, H 5.25, N 11.52; found C 70.63, H 5.01, N 11.41.

\section{$3.8(E)-3 b$-苯基- $13^{1}$ - $\beta, \beta$-二氰亚甲基-20-甲酰乙烯基-} $13^{1}$-去氧焦脱镁叶绿酸-b 甲酯镍配合物(12)的合成

$0{ }^{\circ} \mathrm{C}$ 下向含有 $21 \mathrm{mg}$ 三氯氧磷 $(0.119 \mathrm{mmol})$ 的反应 瓶中滴加 $10 \mathrm{mg} N, N$-二甲基甲酰胺(0.114 mmol), 保持 相同的温度搅拌反应 $15 \mathrm{~min}$, 搅拌下将所得反应溶液加 入到含有 $80 \mathrm{mg}$ 镍配合物 $11(0.110 \mathrm{~mol})$ 的 $0{ }^{\circ} \mathrm{C}$ 二氯甲 烷溶液 $(20 \mathrm{~mL})$ 中, 撤去冰水浴并室温摚拌 $18 \mathrm{~h}$ 后, 加入 $20 \mathrm{~mL}$ 饱和的碳酸钠溶液, 再继续搅拌 $2 \mathrm{~h}$, 用二氯甲烷 萃取反应液 $(20 \mathrm{~mL} \times 3)$, 合并有机相, 用无水硫酸钠干 燥后经重氮甲烷甲基化，除去溶剂，将剩余物通过柱层 析分离 [洗脱剂: $V$ (乙酸乙酯) $: V$ (正己烷 $)=1: 4]$ 得 23 $\mathrm{mg}$ 亮绿色固体 12 (0.029 mmol, 24\%). m.p. 236 239 ${ }^{\circ} \mathrm{C}$; UV-Vis $\left(\mathrm{CHCl}_{3}\right) \lambda_{\max }$ (relative intensity): 409 (1.00), 431 (0.97), 672 (0.31), 738 (0.82) nm; ${ }^{1} \mathrm{H}$ NMR (400 MHz, $\left.\mathrm{CDCl}_{3}\right) \delta: 1.21\left(\mathrm{~d}, J=7.1 \mathrm{~Hz}, 3 \mathrm{H}, 18-\mathrm{CH}_{3}\right), 1.50(\mathrm{t}, J=7.2$ $\mathrm{Hz}, 3 \mathrm{H}, 8-\mathrm{CH}_{3}$ ), $2.00 \sim 2.25,2.30 \sim 2.45,2.58 \sim 2.78$ (each $\mathrm{m}$, all $4 \mathrm{H}, 17 \mathrm{a}+17 \mathrm{~b}-\mathrm{H}$ ), 2.89, 2.94, 3.50, 3.69 (each s, each $3 \mathrm{H}, \mathrm{CH}_{3}+\mathrm{OCH}_{3}$ ), 3.57 (q, $J=7.6 \mathrm{~Hz}, 2 \mathrm{H}, 8 \mathrm{a}-\mathrm{H}$ ), 4.41 (q, $J=7.1 \mathrm{~Hz}, 1 \mathrm{H}, 17-\mathrm{H}), 4.84$ (d, $J=8.6 \mathrm{~Hz}, 1 \mathrm{H}$, $17-\mathrm{H}), 4.80$ (d, $\left.J=19.0 \mathrm{~Hz}, 1 \mathrm{H}, 13^{2}-\mathrm{H}\right), 5.15$ (d, $J=19.0$ $\left.\mathrm{Hz}, 1 \mathrm{H}, 13^{2}-\mathrm{H}\right), 5.88$ (dd, $\left.J=16.0,7.8 \mathrm{~Hz}, 1 \mathrm{H}, 20 \mathrm{~b}-\mathrm{H}\right)$, $7.15(\mathrm{~d}, J=16.8 \mathrm{~Hz}, 1 \mathrm{H}, 3 \mathrm{~b}-\mathrm{H}), 7.30 \sim 7.60(\mathrm{~m}, 4 \mathrm{H}$, $\mathrm{Ph}-\mathrm{H}), 7.74$ (d, $J=6.7 \mathrm{~Hz}, 2 \mathrm{H}, \mathrm{Ph}-\mathrm{H}), 7.84$ (d, $J=16.8 \mathrm{~Hz}$, $1 \mathrm{H}, 3 \mathrm{a}-\mathrm{H}), 8.40$ (d, $J=16.0 \mathrm{~Hz}, 1 \mathrm{H}, 20 \mathrm{a}-\mathrm{H}), 8.90,8.75$ (each s, each 1H, meso-H), 9.89 (d, $J=7.8 \mathrm{~Hz}, 1 \mathrm{H}$, 20c-H); IR (KBr) v: $3323(\mathrm{~N}-\mathrm{H}), 2953(\mathrm{C}-\mathrm{H}), 1735 \sim$ $1697(\mathrm{C}=\mathrm{O}) 1605(\mathrm{C}=\mathrm{C}), 1510$ (chlorin skeleton), 1459, 1444, 1264, 1181, 1078, $1040 \mathrm{~cm}^{-1}$. Anal. calcd for $\mathrm{C}_{46} \mathrm{H}_{40} \mathrm{~N}_{6} \mathrm{NiO}_{3}$ : C 70.51, H 5.15, N 10.73; found C 70.68, H 5.27, N 11.01 . 


\section{$3.913^{2}$-氧代- $13^{1}-\beta, \beta$-二氰亚甲基- $13^{1}$-去氧焦脱镁叶} 绿酸-a 甲酯(14)的合成

将 $50 \mathrm{mg}$ 二氢卟吩二酮 $\mathbf{1 3}(0.089 \mathrm{mmol}), 70 \mathrm{mg}$ 丙二 腈溶解于 $15 \mathrm{~mL}$ 四氢呋喃中, 再加入 $0.2 \mathrm{~mL}$ 三乙胺, 室 温摚拌反应, 约 $10 \mathrm{~min}$ 停止反应. 向反应体系加入 4 $\mathrm{mL}$ 乙酸, 再加入 $20 \mathrm{~mL}$ 水和 $45 \mathrm{~mL}$ 二氯甲烷, 分出有 机层, 用二氯甲烷萃取水相 $(15 \mathrm{~mL} \times 3)$, 合并有机相, 无水硫酸钠干燥, 除去溶剂后硅胶柱层析 [ 展开剂: $V($ 苯 $): V($ 丙酮 $)=10: 1]$, 得 $23 \mathrm{mg}$ 红色固体 $14(0.038$ $\mathrm{mmol}$ ), 产率 43\%. m.p. $213 \sim 216{ }^{\circ} \mathrm{C}$; UV-Vis $\left(\mathrm{CHCl}_{3}\right)$ $\lambda_{\max }$ (relative intensity): 397 (1.00), 483 (0.26), 553 (0.09), 654 (0.18), $716(0.34) \mathrm{nm} ;{ }^{1} \mathrm{H} \mathrm{NMR}\left(\mathrm{CDCl}_{3}\right) \delta:-1.98$ (br s, $1 \mathrm{H}, \mathrm{NH}), 0.51$ (br s, $1 \mathrm{H}, \mathrm{NH}), 1.70(\mathrm{t}, J=7.6 \mathrm{~Hz}, 3 \mathrm{H}$, $\left.8-\mathrm{CH}_{3}\right), 1.88\left(\mathrm{~d}, J=7.3 \mathrm{~Hz}, 3 \mathrm{H}, 18-\mathrm{CH}_{3}\right), 1.95 \sim 2.07$, $2.18 \sim 2.43,2.67 \sim 2.80$ (each $\mathrm{m}$, all $4 \mathrm{H}, 17 \mathrm{a}+17 \mathrm{~b}-\mathrm{H}$ ), $3.35,3.50,3.51,3.62$ (each s, each $3 \mathrm{H}, \mathrm{CH}_{3}+\mathrm{OCH}_{3}$ ), 3.75 (q, $J=7.6 \mathrm{~Hz}, 2 \mathrm{H}, 8 \mathrm{a}-\mathrm{H}), 5.11(\mathrm{~d}, J=8.0 \mathrm{~Hz}, 1 \mathrm{H}, 17-\mathrm{H})$, 4.63 (q, $J=7.5 \mathrm{~Hz}, 1 \mathrm{H}, 18-\mathrm{H}), 6.36(\mathrm{~d}, J=17.8 \mathrm{~Hz}, 1 \mathrm{H}$, $3 \mathrm{~b}-\mathrm{H}$ ), 6.30 (dd, $J=11.5,1.0 \mathrm{~Hz}, 1 \mathrm{H}, 3 \mathrm{~b}-\mathrm{H}), 8.08$ (dd, $J=$ $17.8,11.5 \mathrm{~Hz}, 3 \mathrm{a}-\mathrm{H}$ ), 8.94, 9.50, 9.80 (each s, each $1 \mathrm{H}$, meso-H); IR (KBr) v: $3429(\mathrm{~N}-\mathrm{H}), 2914(\mathrm{C}-\mathrm{H}), 1742 \sim$ $1700(\mathrm{C}=\mathrm{O}), 1604(\mathrm{C}=\mathrm{C}), 1529$ (chlorin skeleton), 1422, 1388, 1304, 1126, 1010, $975 \mathrm{~cm}^{-1}$. Anal. calcd for $\mathrm{C}_{37} \mathrm{H}_{34} \mathrm{~N}_{6} \mathrm{O}_{3}$ : C 72.77, H 5.61, N 13.76; found C 72.61, H 5.41, N 13.59.

\section{$3.1013^{2}$-氧代- $13^{1}-\beta, \beta$-二氭亚甲基- 20 -氯- $13^{1}$-去氧焦 脱镁叶绿酸- $a$ 甲酯(15)的合成}

将 $142 \mathrm{mg}$ 的 $14(0.233 \mathrm{mmol})$ 溶解于 $20 \mathrm{~mL}$ 二氯甲 烷中, 室温条件下缓慢滴入溶有 $110 \mathrm{mg} N$-氯代丁二酰 亚胺(NCS)的 $8 \mathrm{~mL}$ 甲醇溶液, 薄层板(TLC)检测反应进 程, 约 $1.5 \mathrm{~h}$ 滴加完毕, 继续反应 $3 \mathrm{~h}$ 后结束, 搅拌下向 反应体系迅速加入 $40 \mathrm{~mL}$ 水, 分出有机层, 用二氯甲烷 萃取水相 $(100 \mathrm{~mL} \times 3)$; 合并有机相, 用无水硫酸钠干 燥, 减压浓缩, 经硅胶柱层析分离[洗脱剂: $V($ 乙酸乙 酯)：V(正己醇 $)=3: 1$ ] 得 $62 \mathrm{mg}$ 暗红色的固体 $(0.096$ mmol), 产率 $41 \%$. m.p. $220 \sim 223{ }^{\circ} \mathrm{C}$; UV-Vis $\left(\mathrm{CHCl}_{3}\right)$ $\lambda_{\max }$ (relative intensity): 382 (0.42), 437 (1.00), $530(0.06)$, 569 (0.18), $726(0.35) \mathrm{nm} ;{ }^{1} \mathrm{H}$ NMR $\left(\mathrm{CDCl}_{3}\right) \delta:-1.53(\mathrm{br}$ s, $1 \mathrm{H}, \mathrm{NH}),-0.11($ br s, $1 \mathrm{H}, \mathrm{NH}), 1.55(\mathrm{~d}, J=7.3 \mathrm{~Hz}$, $\left.3 \mathrm{H}, 18-\mathrm{CH}_{3}\right), 1.66\left(\mathrm{t}, J=7.6 \mathrm{~Hz}, 3 \mathrm{H}, 8-\mathrm{CH}_{3}\right), 1.92 \sim 2.06$, $2.28 \sim 2.42,2.46 \sim 2.56,2.72 \sim 2.82$ (each $\mathrm{m}$, all $4 \mathrm{H}$, $17 \mathrm{a}+17 \mathrm{~b}-\mathrm{H}), 3.62(\mathrm{q}, J=7.6 \mathrm{~Hz}, 2 \mathrm{H}, 8 \mathrm{a}-\mathrm{H}), 3.16,3.50$, 3.593 .78 (each s, each $3 \mathrm{H}, \mathrm{OCH}_{3}+\mathrm{CH}_{3}$ ), 4.81 (q, $J=7.1$ $\mathrm{Hz}, 1 \mathrm{H}, 18-\mathrm{H}), 5.20$ (d, $J=9.2,2.7 \mathrm{~Hz}, 1 \mathrm{H}, 17-\mathrm{H}), 6.13$ (dd, $J=17.8,1.4 \mathrm{~Hz}, 1 \mathrm{H}$, trans-3b-H), 6.27 (dd, $J=11.5$,
$1.4 \mathrm{~Hz}, 1 \mathrm{H}$, cis-3b-H), 7.82 (dd, $J=17.8,11.5 \mathrm{~Hz}, 1 \mathrm{H}$, 3a-H), 9.52, 9.58 (each s, each $1 \mathrm{H}$, meso-H); IR (KBr) $v$ : $3410(\mathrm{~N}-\mathrm{H}), 2938(\mathrm{C}-\mathrm{H}), 1687 \sim 1731(\mathrm{C}=\mathrm{O}), 1622$ $(\mathrm{C}=\mathrm{C}), 1554$ (chlorin skeleton), 1436, 1228, 1162, 1040, $985 \mathrm{~cm}^{-1}$. Anal. calcd for $\mathrm{C}_{37} \mathrm{H}_{33} \mathrm{ClN}_{6} \mathrm{O}_{3}: \mathrm{C} 68.88, \mathrm{H} 5.16$, N 13.03; found C 68.65, H 5.29, N 13.11.

\section{References}

[1] Chen, Y.-H.; Sumlin, A.; Morgan, J.; Gryshuk, A.; Oseroff, A.; Henderson, B. W.; Dougherty, T. J.; Pandey, R. K. J. Med. Chem. 2004, 47, 4814.

[2] Nyman, E. S.; Hynninen, P. H. J. Photochem. Photobiol. B 2004, $73,1$.

[3] Ethirajan, M.; Joshi, P.; William, W. H.; Ohkubo, K.; Fukuzumi, S.; Pandey, R. K. Org. Lett. 2011, 8, 1956

[4] (a) Chen, Y. H.; Li, G. L.; Pandey, R. K. Curr. Org. Chem. 2004, 8, 1105 .

(b) Bellnier, D. A.; Greco, W. R.; Loewen, G. M.; Nava, H.; Oseroff, A. R.; Pandey, R. K.; Tsuchida, T.; Dougherty, T. J. Cancer Res. 2003, 63, 1806.

[5] (a) Steruberg, E. D.; Dolphin, D. Tetrahedron 1998, 54, 4151.

(b) Washington, I.; Brooks, C.; Turro, N. J.; Nakanishi, K. J. Am. Chem. Soc. 2004, 126, 9892.

[6] (a) Mennenga, A.; Wolfgang Gartner, W.; Lubitz, W.; Gorner, H Phys. Chem. Chem. Phys. 2006, 8, 5444.

(b) Ethirajan, M.; Joshi, P.; William, W. H.; Ohkubo, K.; Fukuzumi, S.; Pandey, R. K. Org. Lett. 2011, 13, 1956.

(c) Wang, J. J.; Li, J.-J.; Wu, X.-R.; Shim, Y.-K. Chin. J. Chem. 2006, 24, 933.

[7] (a) Li, J.-Z.; Wang, J.-J.; Yoon, L.; Cui, B.-C.; Shim, Y.-K. Bioorg Med. Chem. Lett. 2012, 22, 1846.

(b) Wang, J. J.; Liu, C.-L.; Li, J.-J. Synth. Commun. 2012, 42, 487.

(c) Wang, J. J.; Wang, P.; Li, J.-J.; Jakus, J.; Shim, Y.-K. Bull. Korean Chem. Soc. 2011, 32, 3473.

[8] (a) Wang, J.-J.; Li, J.-Z.; Li, Y.-W.; Jakus, J.; Shim, Y.-K. J. Porphyrins Phthalocyanines 2010, 14, 860.

(b) Wang, J.-J.; Han, G.-F.; Shim, Y.-K. J. Iran Chem. Soc. 2011, $8,965$.

[9] Wu, X.-R.; Liu, C.; Yang, Z.; Yao, N.-N.; Wang, J.-J. Chin. J. Org. Chem. 2012, 32, 632 (in Chinese).

(邬旭然, 刘超, 杨泽, 姚楠楠, 王进军, 有机化学, 2012, 32, 632.)

[10] Wang, P.; Yang, Z.; Li, J. Z.; Yao, N.-N.; Wang, J.-J. Chin. J. Org. Chem. 2012, 32, 368 (in Chinese).

(王朋, 杨泽, 李家柱, 姚楠楠, 王进军, 有机化学, 2012, 32, 368.)

[11] Wang, J.-J.; Zhao, L.-L.; Li, J.-Z. Chin. J. Org. Chem. 2009, 29, 1958 (in Chinese).

(王进军, 赵丽丽, 李家柱, 有机化学, 2009, 29, 1958.)

[12] Wang, J.-J. Chin. J. Org. Chem. 2005, 25, 1353 (in Chinese). (王进军, 有机化学, 2005, 25, 1353.)

[13] Liu, C.; Dobhal, M. P.; Ethirajan, M.; Missert, J. R.; Pandey, R. K.; Balasubramanian, S.; Sukumaran, D. K.; Zhang, M.; Kadish, K. M.; Ohkubo, Fukuzumi, K. S. J. Am. Chem. Soc. 2008, 130, 14311.

[14] (a) Li, J.-Z. M. S. Thesis, Yantai University, Yantai, 2007 (in Chinese).

(李家柱, 硕士论文, 烟台大学, 烟台, 2007.)

(b) Wang, J.-J.; Han, G.-F.; Yin, J.-G.; Wu, X.-R.; Zhao, Y.; Gong, B.-A.; Shim, R.-K. Acta Chim. Sinica 2003, 61, 907. 
(王进军, 韩光范, 殷军港, 鸟旭然, 赵岩, 宫宝安, 沈荣基, 化 学学报, 2003, 61, 907.)

[15] Wu, J.; Yin, J.-G.; Zhang, Q.; Sun, C.-M.; Li, F.-G.; Pei, W.; Wang, J.-J. Chin. J. Org. Chem. 2011, 31, 1653 (in Chinese).

(武进, 殷军港, 张千, 孙传民, 李付国, 裴文, 王进军, 有机化 学, 2011, 31, 1653.)

[16] Smith, K. M.; Gogg, D. A.; Simpson, D. J. J. Am. Chem. Soc. 1985, 107, 4946.
[17] Li, J.-Z.; Liu, W.-H.; Li, F.-G.; Wang, J.-J.; Suo, Y.-R.; Liu, Y.-J. Chin. J. Org. Chem. 2007, 27, 1594 (in Chinese).

(李家柱, 刘万卉, 李付国, 王进军, 索有瑞, 刘永军, 有机化学, 2007, 27, 1594.)

[18] Tamiaki, H.; Kouraba, M. Tetrahedron 1997, 53, 10677.

[19] Shin-ichi Sasaki, S.; Yoshizato, M.; Kunieda, M.; Tamiaki, H. Eur. J. Org. Chem. 2010, 5287.

(Li, L.; Lu, Z.) 